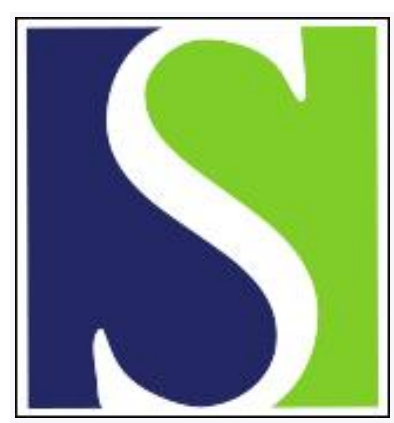

Scand J Work Environ Health 1992;18(6):408-409

https://doi.org/10.5271/sjweh.1555

Issue date: 01 Dec 1992

On classifying reproductive studies according to study design and using odds ratios in the presentation of results.

by Nurminen $T$

This article in PubMed: www.ncbi.nlm.nih.gov/pubmed/1485167

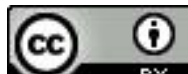


Scand J Work Environ Health 1992;18:408-9

\section{On classifying reproductive studies according to study design and using odds ratios in the presentation of results}

I would like to comment on some methodological aspects of the recent review by Dr Marian C Marbury (1). First, classifying epidemiologic reproductive studies on the basis of certain features of study design arouses a need for contemplation. Second, the quantitative results of the reviewed studies were presented in a way that can lead to biased interpretations.

In her review, Dr Marbury classified epidemiologic studies on the effects of ergonomic factors on birthweight and gestational age according to the adopted study design and labeled them as either cross-sectional, case-referent, prospective cohort, or prospective (table 1 in reference 1). Her definition of cross-sectional design was as follows: "In the cross-sectional design, women are interviewed soon after delivery about a wide variety of exposures that occurred before or during pregnancy or both [ $p$ 74]." When considering this definition, one is left pondering what the cross-sectional aspect was that differentiated these studies from the others.

In the design of epidemiologic studies, one of the key concerns is to define the time period during which one is to ascertain the outcome information, that is, whether one is to observe the base population's experience over time (either prospectively or retrospectively) or the study outcome in a population crosssection with respect to time (2). A further aspect of the design is whether the studied relation between exposure and outcome is cross-sectional or longitudinal (2). Commonly a contemporaneous observation of the base population with respect to both exposure and outcome is thought to be the essence of the cross-sectional design (3). The meaningfulness of a cross-sectional relation is assessed according to the duration of the process that connects the outcome with the exposure, the stability of the exposure, and, also, any influence of the outcome on the exposure (2).

In all reproductive studies the proportion of babies born preterm or with a low birthweight is by definition calculated from information observed at birth. In addition the occurrence of babies small for their gestational age has, in all studies, been observed in crosssections of newborns, and no means have been available with which to identify cases of intrauterine growth retardation in fetuses before birth. Thus the studies reviewed by Dr Marbury did not differ with respect to the time period during which the outcomes were considered, and all of them observed newborns. Incidentally, this practice has also been unavoidable in studies on congenital malformations in that such studies have not been able to include cases that have been lost through early embryonic or fetal death, and they thus have usually had to be satisfied with observing only newborns.

According to Dr Marbury's description cross-sectional studies consider " ... exposures that occurred before or during pregnancy or both [p 74]" (1). Thus these studies can examine longitudinal relations with respect to the concerned outcomes observed at birth. Accordingly, all the "cross-sectional" studies listed in table 1 of Dr Marbury's article indeed attempted to examine longitudinal relations, as did also all of the other studies reviewed.

Real success in relating an outcome to an exposure with reference to a time period that might be etiologically relevant presumes an understanding of the process that could connect the two and, also, the accuracy of their measurement. In Dr Marbury's description of a "cross-sectional" study, the only characteristic was the retrospective acquisition of exposure information. In this situation, possibilities to improve the accuracy of the measurement of exposures are limited when compared with the means available in prospective studies, as was pointed out in the article (1). However, contrary to the reader's expectations, the so-labeled crosssectional studies examining outcomes at birth did not seem to have any cross-sectional features that would have differentiated them from the reviewed prospective studies.

A further concern to the reader of the article is the presentation of odds ratios in table 1 , calculated by Dr Marbury from the original data. The reader can easily make biased interpretations when considering odds ratios instead of the actual ratios of proportions of adverse outcomes in the compared exposure groups (risk ratios). For example, the crude "prevalence odds ratio calculated from the data [p 77]" (1) of one study (4) was 2.2. However, interpreting the risk as 2.2 -fold would be an exaggeration because the crude risk ratio presented in the original article was, in fact, 1.5. Note, too, that, in the text of Dr Marbury's review, the point estimate of the corresponding adjusted risk ratio, 1.4, from the original article was erroneously presented as an odds ratio of 1.4 .

In the case-referent approach with sampling of the noncase segment of a study base, one calculates (casereferent) exposure odds ratios from the data as an approximation for risk ratios. An unbiased estimation presupposes that the "rare-disease assumption" is satisfied. However, when the study data provide for a straightforward estimation of risk ratios, one may question the practice of presenting results referring to 
ratios of risks for adverse outcomes in terms of disease odds ratios. Appropriate developments in data analytical tools for binomial regression (eg, reference 5) enable intelligible reporting of research results (6) also when possible confounders are controlled.

\section{References}

1. Marbury MC. Relationship of ergonomic stressors to birthweight and gestational age. Scand J Work Environ Health 1992;18:73-83.

2. Miettinen OS. Theoretical epidemiology: principles of occurrence research in medicine. Albany, NY: Delmar Publishers Inc, 1985.

3. Rothman NJ. Modern epidemiology. Boston, MA: Little, Brown and Company, 1986.

4. Nurminen T, Lusa S, Ilmarinen J, Kurppa K. Physical work load, fetal development and course of pregnancy. Scand J Work Environ Health 1989;15:404-14.

5. Wacholder S. Binomial regression in GLIM: estimating risk ratios and risk differences. Am J Epidemiol 1986; 123:174-84.

6. Greenland $S$. Interpretation and choice of effect measures in epidemiologic analyses. Am J Epidemiol 1987;125: $761-8$

Tuula Nurminen, DrPH

Department of Epidemiology and Biostatistics

Institute of Occupational Health

Topeliuksenkatu 41 a $\mathrm{A}$

SF-00250 Helsinki Finland

\section{Author's reply}

I thank Dr Nurminen for her thoughtful response (1) to my article (2). I would agree that the studies I have described as cross-sectional are indeed longitudinal in nature, inasmuch as information on exposures during pregnancy, not at delivery, was ascertained. On further reflection concerning what distinguishes these studies, which I will refer to as newborn investigations, from more traditional cohort studies, I realize that the essence of the difference is in the conceptualization of the study base.

In any study design, a base population is identified, cases are ascertained, and information about the base is obtained on a sample or census basis (3). In a traditional prospective cohort study one would assemble a cohort of women who were early in their pregnancy, and some of whom were exposed to the determinant of interest. They would be followed to the end of their pregnancy and rates of low birthweight and prematurity would be calculated. The study base would be women who are pregnant. In contrast, the implied study base in an investigation of newborns is all women who become pregnant and do not have a spontaneous or induced abortion. Given the limitations of current research tools, particularly the inability to identify all pregnancies and to detect intrauterine retardation in aborted fetuses, this distinction may be of limited practical importance. However, as rates of spontaneous and induced abortion may differ among exposure groups and as adverse reproductive outcomes may be interrelated (for example, a high dose of a toxin may result in a spontaneous abortion, while a lower dose results in intrauterine growth retardation), newborn investigations may produce a somewhat distorted picture of the reproductive effects of a particular exposure. Case-referent studies, which focus on one outcome and which usually sample from the same base population as newborn investigations, share this limitation. In summary, then, a newborn investigation may be thought of as a cohort study in which admittance to the cohort is conditional on fetal survival to the age when delivery takes place in a hospital.

As for the parameter of interest in these studies, it appears to differ between intrauterine growth retardation and premature birth. If all premature births are ascertained, including those that are stillborn, the cumulative incidence and the risk ratio can be directly calculated, as Dr Nurminen states (1). However, as Dr Nurminen also points out, epidemiologists have not been able to identify cases of intrauterine growth retardation in fetuses before birth. This may be a more important limitation for congenital malformations. However, if it is an important limitation, then it follows that the prevalence of this outcome is being measured at birth. Thus the risk ratio calculated by Dr Nurminen and her colleagues (4) is in fact a prevalence rate ratio.

\section{References}

1. Nurminen T. On classifying reproductive studies according to study design and using odds ratios in the presentation of results. Scand J Work Environ Health 1992;18: 408-9.

2. Marbury MC. Relationship of ergonomic stressors to birthweight and gestational age. Scand $\mathbf{J}$ Work Environ Health 1992;18:73-83.

3. Miettinen OS. Theoretical epidemiology: principles of occurrence research in medicine. Albany, NY: Delmar Publishers Inc, 1985.

4. Nurminen T, Lusa S, Ilmarinen J, Kurppa K. Physical work load, fetal development and course of pregnancy. Scand J Work Environ Health 1989;15:404-14.

Marian C Marbury, ScD

Minnesota Department of Health

717 SE Delaware St

Minneapolis, MN 55414 USA 